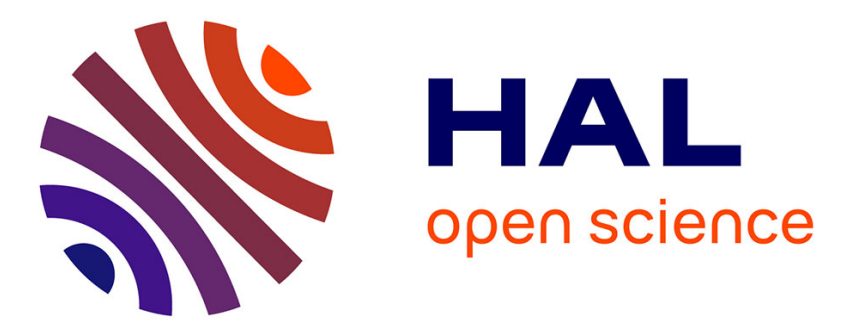

\title{
On the precision in polyhedral partition representation and the fragility of PWA control
}

Rajesh Koduri, Sorin Olaru, Pedro Rodriguez-Ayerbe

\section{To cite this version:}

Rajesh Koduri, Sorin Olaru, Pedro Rodriguez-Ayerbe. On the precision in polyhedral partition representation and the fragility of PWA control. 56th IEEE Conference on Decision and Control (CDC 2017), Dec 2017, Melbourne, Australia. 10.1109/cdc.2017.8264026 . hal-01648012

\section{HAL Id: hal-01648012 \\ https://hal-centralesupelec.archives-ouvertes.fr/hal-01648012}

Submitted on 24 Nov 2017

HAL is a multi-disciplinary open access archive for the deposit and dissemination of scientific research documents, whether they are published or not. The documents may come from teaching and research institutions in France or abroad, or from public or private research centers.
L'archive ouverte pluridisciplinaire HAL, est destinée au dépôt et à la diffusion de documents scientifiques de niveau recherche, publiés ou non, émanant des établissements d'enseignement et de recherche français ou étrangers, des laboratoires publics ou privés. 


\title{
On the precision in polyhedral partition representation and the fragility of PWA control
}

\author{
Rajesh Koduri ${ }^{1}$ Sorin Olaru ${ }^{1}$ and Pedro Rodriguez-Ayerbe ${ }^{1}$
}

\begin{abstract}
Explicit model predictive control (EMPC) solves a multi-parametric Quadratic Programming (mp-QP) problem for a class of discrete-time linear system with linear inequality constraints. The solution of the EMPC problem in general is a piecewise affine control function defined over non-overlapping convex polyhedral regions composing a polyhedral partition of the feasible region. In this work, we consider the problem of perturbations on the representation of the vertices of the polyhedral partition.Such perturbations may affect some of the structural characteristics of the PWA controller such as "non-overlapping within the regions" or "the closed-loop invariance". We first show how a perturbation affects the polyhedral regions and evoke the overlapping within the modified polyhedral regions. The major contribution of this work is to analyze to what extend the non-overlapping and the invariance characteristics of the PWA controller can be preserved when the perturbation takes place on the vertex representation. We determine a set called sensitivity margin to characterize for admissible perturbation preserving the non-overlapping and the invariance property of the controller. Finally, we show how to perturb multiple vertices sequentially and reconfigure the entire polyhedral partition.
\end{abstract}

\section{INTRODUCTION}

Explicit control laws can be efficiently implemented on hardware circuits for systems with fast dynamics and relatively small dimension of system states [1]-[3]. Recently, such control laws have gained popularity for a wide range of realtime control applications [4]-[6]. However, the adoption of such control laws are pertained to the numbers of state space partitions and the piecewise affine control laws associated with those partitions. In order to exploit the computational advantages of the explicit controller, a truncation or quantization operation must be performed on the coefficients of the representation of the state space partitions and on their associated PWA controls [7]. The implications of the quantized state-partitions and the quantized PWA gains and offsets extend to affect control input accuracy, whose computations are based on point location functions, and the properties of the PWA controller. The quantized state partitions might also adversely affect the non-overlapping (uniqueness) and nonemptiness (feasibility) characteristics of the PWA controller. The authors of [8] and [9] proposed a geometrical approach to determine robustness/fragility margins with respect to the invariance characteristics of the PWA controller. However

\footnotetext{
*The work leading to these results has received funding from the People Programme (Marie Curie Actions) of the European Unions Seventh Framework Programme (FP7/2007-2013) under REA grant agreement no 607957 (TEMPO).

${ }^{1}$ The Authors are with L2S, CentraleSupelec-CNRSUPS, Paris Saclay University, 91192, Gif-Sur-Yvette, France. rajesh.koduri; pedro.rodriguez-ayerbe; sorin.olaru@centralesupelec.fr
}

their approach does not extend to the quantized representation of the state space partitions. In a recent study [10], the accuracy of the explicit control input for the quantized representation of the regions and the quantized representation of the PWA control laws are analyzed in general to prove the scale of quantization required in order to obtain a certain degree of control accuracy. However, the paper builds the control input analysis on the assumption that the modified state space regions are non-overlapping and thus does not address one of the essential characteristics of the representation of the state partitions: the non-overlapping and completeness of the polyhedral partition of the feasible domain.

This paper is a continuation of earlier work [11] where a vertex sensitivity region is derived that guarantees the nonoverlapping characteristics of the PWA controller in the event of perturbation on the vertex representation of the partition. In this work we extend the above results to analyze to what extend the non-overlapping and the invariance characteristics of the PWA controller can be preserved when a perturbation on the vertex representation of the partition takes place. First, we approximate the outer representation of the feasible set for admissible perturbation on the frontier vertices, which are considered to be sensitive all by ensuring the feasible set to preserve the non-overlapping and the invariance property of the controller. Second, starting from the new feasible domain, we analyze the polyhedral partitions by considering each of the vertices of the partition which are not placed on the frontiers of the feasible domain. We determine a set called sensitivity margin for the closed-loop invariance. This set characterizes for admissible perturbation on the vertex representation to preserve the non-overlapping and the invariance characteristics of the PWA controller. Third, we compute the perturbed polyhedral partitions for all the inner vertices of the feasible set sequentially completing the transformation of the original polyhedral regions to a new polyhedral regions for all the vertices in the feasible set.

\section{BASIC NOTATIONS}

This section addresses some basic notations and definitions. The sets $\mathbb{R}, \mathbb{Z}, \mathbb{N}$ and $\mathbb{N}_{+}$denote set of real numbers, set of integers, set of non-negative integers and set of positive integers respectively. A vector is noted $x \in \mathbb{R}^{n}, x=[$ $\left.x_{1}, \cdots, x_{n}\right]^{T}$ and a matrix $S \in \mathbb{R}^{n \times m}, S=\left[s_{i j}\right]$. An identity matrix is represented by $I_{n}$, where the subscript $n$ denotes the dimension of that matrix.

A polyhedron is the (convex) intersection of a finite number of open or closed half-spaces and a polytope is a bounded and closed polyhedron. For a $N \in \mathbb{N}_{+}, \mathcal{I}_{N}$ denotes 
the set of integers, $\mathcal{I}_{N}:=\left\{i \in \mathbb{N}_{+} \mid i \leq N\right\}$. For a given set $\mathcal{P}, \operatorname{int}(\mathcal{P})$ denotes the interior of $\mathcal{P}$ and Conv denotes the convex hull. The set of vertices of a polytope $\mathcal{P} \subset \mathbb{R}^{n}$ is denoted $\mathcal{V}(\mathcal{P})$. Given a matrix $S, \mathcal{V}(S)$ denotes the set of points described by the colums of $S$.

\section{SySTEM DESCRIPTION}

Consider a linear discrete-time system given by,

$$
x_{k+1}=A x_{k}+B u_{k}
$$

here, $x_{k} \in \mathbb{R}^{n}$ and $u_{k} \in \mathbb{R}^{m}$ denote the state vector and input variables respectively at time $k$.

The constraints on the system's states and input variables are represented by polytopic sets,

$$
\begin{array}{r}
\mathcal{X}=\left\{x: H_{x} x \leq h_{x}, \quad H_{x} \in \mathbb{R}^{n_{x} \times n}, h_{x} \in \mathbb{R}^{n_{x}}\right\} \\
\mathcal{U}=\left\{u: H_{u} u \leq h_{u}, \quad H_{u} \in \mathbb{R}^{m_{u} \times m}, h_{u} \in \mathbb{R}^{m_{u}}\right\}
\end{array}
$$

The constrained control law is considered to be designed upon a classical MPC framework for (1)-(2) with a quadratic cost function and standard stability guarantees based on the terminal costs and terminal constraints [12]. Subsequently the receding horizon constrained problem is transformed into a multi-parametric Quadratic Programming (mp-QP) problem [2] to obtain a so-called explicit MPC (EMPC) formulation for the feedback control law.

The solution of EMPC problem is a finite set of affine functions defined over the polyhedral partition of the set $\mathcal{R}$, where $\mathcal{P}_{N}(\mathcal{R})=\left[\mathcal{R}_{1}, \mathcal{R}_{2} \cdots \mathcal{R}_{N}\right]$ also called polyhedral partitions of the set $\mathcal{R}$. The polyhedral regions $\mathcal{R}_{i}$ are nonoverlapping such that $\operatorname{int}\left(\mathcal{R}_{i}\right) \cap \operatorname{int}\left(\mathcal{R}_{j}\right)=\emptyset$, for $i \neq j$. The non-overlapping property ensures the unique solution for the point location of a given state $x_{k} \in \mathcal{R}_{i}, \forall i \in \mathcal{I}_{N}$. Two neighboring polyhedral $\mathcal{R}_{i}$ and $\mathcal{R}_{j}$ share common facets. It is worth to be recalled that the feasible set $\mathcal{R}$ represents a bounded polyhedron in itself.

Definition 1: A mapping from $\mathbb{R}^{n}$ to $\mathbb{R}^{m}$ described by:

$$
f_{\text {pwa }}(x)=F_{i} x+g_{i}, \forall x \in \mathcal{R}_{i}, i \in \mathcal{I}_{N}
$$

is called a piecewise affine function over $\cup_{i \in \mathcal{I}_{N}} \mathcal{R}_{i}$.

The EMPC feedback law is given in terms of the piecewise affine function defined over the polyhedral partition $\mathcal{P}_{N}(\mathcal{R})$ of the set $\mathcal{R} \subset \mathbb{R}^{n}$ and it can be written as:

$$
u_{\text {pwa }}\left(x_{k}\right)=F_{i} x_{k}+g_{i}, \quad \forall x_{k} \in \mathcal{R}_{i} .
$$

Definition 2: The closed and bounded set $\mathcal{R} \subset \mathcal{X}$ is called positively invariant with respect to the system (1) in closed loop with the control law $u_{p w a}\left(x_{k}\right)$ in (4) if $\forall x(0) \in \mathcal{R}$, it follows $x_{k} \in \mathcal{R}, \forall k \in \mathbb{Z}_{[1 ; \infty]}$.

By construction of the standard MPC the positive invariance is considered to be guaranteed for the explicit MPC law.

\section{Motivation and Problem Formulation}

For real-time implementation of explicit controller, the online implementation of the PWA control law is composed of three stages,

(i) Storage of the polyhedral regions $\mathcal{R}_{i}$, the PWA control gains $F_{i}$ and affine components $g_{i}$. (ii) Implementation of a point location mechanism with respect to the parameter $x$ and the polyhedral partitions $\mathcal{R}=\cup_{i=1}^{N} \mathcal{R}_{i}$.

(iii) On-line evaluation of the PWA control law $u_{\text {pwa }}(x)=$ $F_{i} x+g_{i}$ based on the elements resulting from the first two stages and the state measurements.

In practice this evaluation procedure can fail due to several reasons. Some of those are i) the precision of $\mathcal{R}_{i}$ representation ii) due to point location mismatch iii) PWA control accuracy inflicted by the precision of representation of the control gain $F_{i}$ and offset $g_{i}$.

The PWA control accuracy and the fragility issues of the $F_{i}$ and $g_{i}$ has been extensively discussed in [8]-[10]. The resulting solution obtained from the EMPC problem is a set of PWA functions defined over the polyhedral partition $\mathcal{P}_{N}(\mathcal{R})$ and their analysis in the point iii) above can be handled in the respective framework. However, the issues related with the representation and the closely related point location problems (items (i and ii) above) remain largely uncovered and will represent the main goal of the present study. Before entering into the details of the main results, let us motivate the chosen approach by considering a polyhedral region $\mathcal{R}_{i} \subset \mathcal{R} \in \mathbb{R}^{n}, i \in \mathcal{I}_{N}$, and its half-space representation is given by,

$$
\mathcal{R}_{i}=\left\{x \mid h_{i, r_{b}} x \leq b_{i, r_{b}}, \forall i \in \mathcal{I}_{N}, \quad r_{b}=1, \cdots, r_{i}\right\}
$$

here, $r_{i}$ denotes the number of closed half-spaces of the region $\mathcal{R}_{i}$. In order to analyze the sensitivity of the polyhedral partition representation and its implication on the PWA control, a perturbation in the representation of the half-space $\left\{h_{i, r_{b}} \leq b_{i, r_{b}}\right\}$, for some $r_{b} \in \mathcal{I}_{r_{i}}$ of the region $\mathcal{R}_{i}$ will be considered,

$$
\hat{h}_{i, r_{b}}=h_{i, r_{b}}+\Delta h_{i, r_{b}} \text { and } \hat{b}_{i, r_{b}}=b_{i, r_{b}}+\Delta b_{i, r_{b}}
$$

which leads to a new polyhedral set:

$$
\hat{\mathcal{R}}_{i}=\left\{x \mid \hat{h}_{i, r_{b}} x \leq \hat{b}_{i, r_{b}}\right\}
$$

The perturbation of the half-space inequalities of the region $\mathcal{R}_{i}$ will concomitantly affect all the neighbor regions $\mathcal{R}_{j}$ sharing the respective frontier within their half-space representation. As several regions are affected, the analysis of the effects on the partition will encounter structural problems.

1) Overlapping of the polyhedral partitions, violating the PWA characterization: $\operatorname{int}\left(\hat{\mathcal{R}}_{i}\right) \cap \operatorname{int}\left(\hat{\mathcal{R}}_{j}\right)=\emptyset, \forall i \neq j$. 2) $\mathcal{R} \backslash \cup_{i=1}^{N} \hat{\mathcal{R}}_{i} \neq \emptyset$ posing an well-possessedness issue in the characterization of the polyhedral partition.

The first type of problem arise from the asymmetric consideration of the perturbation in between neighboring regions while the second can take place even if the perturbation is treated similarly among the neighboring regions. Moreover, both phenomena lead to invalidation of the PWA control law defined over the partitions. Particularly the second phenomenon leaves the point location function seemingly untraceable and this case is shown in Figure 1. The drawbacks demonstrated by the perturbation on the half-space representation are the consequence that the perturbations are not considered 


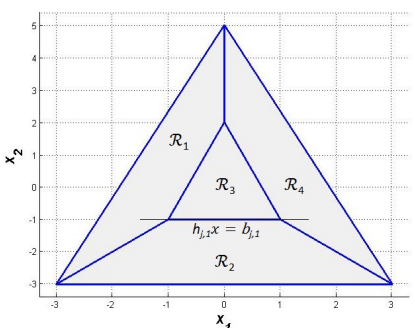

(a) 2-D Polyhedral with four (a) 2-D Illustration of regions $\hat{\mathcal{R}}_{1}$, regions $\mathcal{R}=\cup_{i=1}^{4} \mathcal{R}_{i}$ before $\hat{\mathcal{R}}_{2}, \hat{\mathcal{R}}_{3}$ and $\hat{\mathcal{R}}_{4}$ after perturbaperturbation of the half-space re- tion. Such a reconfiguration propresentation. duces holes in the feasible domain that will undermine the wellpossessedness characteristics.

Fig. 1: 2-D polyhedral representation before and after perturbation on the half-space representation

jointly for different half-spaces. This is due to the fact that the closed half-spaces of the regions $\mathcal{R}_{i}$ are described uniquely and addressing perturbation on such representation is not possible locally. These drawbacks force us to rely on the duality of the polyhedron and lead us to the following problem formulation. $\mathrm{Eq} \mathrm{(6)} \mathrm{can} \mathrm{be} \mathrm{given} \mathrm{with} \mathrm{equivalent}$ vertex representation in the virtue of Motzkin duality:

$$
\mathcal{R}_{i}=\operatorname{Conv}\left\{v_{i, 1}, \cdots, v_{i, r_{i}}\right\}, \forall i \in \mathcal{I}_{N}
$$

here $r_{i}$ is the number of vertices of $\mathcal{R}_{i}$. Now, consider a perturbation with respect to the vertex representation $v_{i, j}, j \in \mathcal{I}_{r_{i}}$ of the region $\mathcal{R}_{i}$,

$$
\hat{v}_{i, j}=v_{i, j}+\Delta v_{i, j}, \quad i \in \mathcal{I}_{N}, j \in \mathcal{I}_{r_{i}}
$$

this will lead to a new polyhedral set:

$$
\hat{\mathcal{R}}_{i}=\operatorname{Conv}\left\{v_{i, 1}+\Delta v_{i, 1}, \cdots, v_{i, r_{i}}+\Delta v_{i, r_{i}}\right\} .
$$

In order to illustrate the representation in this framework and present the obvious advantages of considering perturbation on the vertex representation a similar partition to the one presented in Figure 1 is depicted in the Figure 2. This time it is obvious that the completeness of the partition is not lost. In general terms, the case $R \backslash \cup_{i=1}^{N} \hat{\mathcal{R}}_{i} \neq \emptyset$ is avoided from the consequences of the perturbations in the polyhedral partition. To resume, the main objective is to start from the existence of the system in the form (1) stabilized by a PWA control law, and discuss the impact of perturbations on the vertex representation of the polyhedral region by proposing:

- An analysis of the admissible perturbations with respect to the non-overlapping and invariance characteristics of the PWA controller.

\section{TREATMENT OF ONE VERTEX CONSIDERED INDEPENDENTLY}

\section{A. Vertex Perturbation with respect to non-overlapping cha-} racterization

In the following, the definition of the vertex sensitivity for a single vertex is provided under the assumption that all the other vertices are fixed and not subject to perturbations.
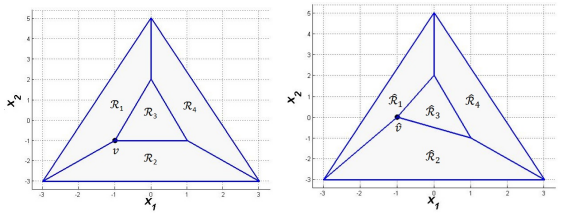

(a) 2-D Polyhedral (b) Regions $\hat{\mathcal{R}}_{1}$, with four regions $\hat{\mathcal{R}}_{2}, \quad \hat{\mathcal{R}}_{3}$ and $\hat{\mathcal{R}}_{4}$ $\mathcal{R}=\cup_{i=1}^{4} \mathcal{R}_{i}$ after perturbation before perturbation preserving of the vertex the property, $\operatorname{nint}\left(\hat{\mathcal{R}}_{j}\right) \neq$ representation. $\quad \operatorname{int}\left(\hat{\mathcal{R}}_{i}\right) \cap \operatorname{int}\left(\mathcal{R}_{j}\right)=\emptyset, \forall i, j \in \mathcal{I}_{4}, i \neq j$. $\emptyset, \forall i, j \in \mathcal{I}_{4}, i \neq j$.

Fig. 2: 2-D polyhedral representation before and after perturbation of the vertex representation

Definition 3: Consider the set of partitions $\mathcal{P}_{N}(\mathcal{R}) \in \mathbb{R}^{n}$ with $\mathcal{R}_{i}=\operatorname{Conv}\left\{v_{i, 1}, \cdots, v_{i, r_{i}}\right\}, i \in \mathcal{I}_{N}$ given by vertex representation. Assume $v \in \mathbb{R}^{n}$ be a vertex within $\mathcal{P}_{N}(\mathcal{R})$ and denote $\Theta^{v}$ as the set of indexes of polyhedral regions having $v$ as a vertex:

$$
\Theta^{v}=\left\{j \in \mathcal{I}_{N} \mid v \in \mathcal{V}\left(\mathcal{R}_{j}\right)\right\}
$$

The set $\Psi^{v} \subset \mathcal{R} \subset \mathbb{R}^{n}$ is describing the vertex sensitivity for $v$ if $\forall(v+\Delta v) \in \Psi^{v}$ the collection of sets

$$
\left\{\begin{array}{l}
\hat{\mathcal{R}}_{j}=\operatorname{Conv}\left\{\mathcal{V}\left(\mathcal{R}_{j}\right) \backslash\{v\}, v+\Delta v\right\}, \forall j \in \Theta^{v}, \\
\hat{\mathcal{R}}_{j}=\mathcal{R}_{j}, \forall j \in \mathcal{I}_{N} \backslash \Theta^{v}
\end{array}\right.
$$

represents a polyhedral partition: $\hat{\mathcal{P}}_{N}(\mathcal{R})=\left\{\hat{\mathcal{R}}_{1}, \cdots, \hat{\mathcal{R}}_{N}\right\}$.

Theorem 1: Consider the subset of regions $\mathcal{R}_{j}, j \in \Theta^{v}$ of $\mathcal{P}_{N}(\mathcal{R})$ such that $v \in \mathcal{V}\left(\mathcal{R}_{j}\right), \forall j \in \Theta^{v}$, then the perturbed vertex sensitivity for $v$ is represented by a polyhedral set.

Proof: For interested readers, please refer proof 1 in [11] .

\section{B. Vertex Perturbation with respect to invariance characte- rization}

In this section, we discuss the invariance characterization of the PWA controller as an additional "dynamics-related" property, to be considered on top of the non-overlapping property of the PWA control. We will preserve the assumption that only one vertex is perturbed at the time. This last point will be relaxed in section VI.

From Theorem 1, it is understood that the vertex sensitivity is analyzed for the admissible perturbation related to the nonoverlapping characteristics for any single vertex that belongs to the polyhedral regions $\mathcal{R}_{i}$. In order to incorporate the analysis for vertex sensitivity and the invariance property of the PWA control law, we will have to deal with the vertices that are sensitive to perturbation. The vertices that represent extreme points of the set $\mathcal{R}$ are particularly sensitive to perturbation taking into account that they characterize the controlled-invariant properties per se. This can result in the invalidation of the invariant set with respect to the nominal system and the constraints given in equation (1) and (2). 
1) Perturbations of extreme points of the feasible domain $\mathcal{R}$ : In the following, we aim to analyze the perturbation of vertices that represent extreme point of the set $\mathcal{R}$ and reconstruct the polyhedral partition $\mathcal{R}=\left\{\mathcal{R}_{1}, \cdots, \mathcal{R}_{N}\right\}$.

Now, we define the set of vertices on the frontier of $\mathcal{R}$ as:

$$
V=\left\{v \in \mathcal{R}: \exists i \text { such that } v \in \mathcal{V}\left(\mathcal{R}_{i}\right) \text { and } v \notin \operatorname{int}(\mathcal{R})\right\}
$$

Consider the set $\mathcal{R}=\cup_{i=1}^{N} \mathcal{R}_{i}$, with $\mathcal{R}_{i}=$ $\operatorname{Conv}\left\{v_{i, 1}, v_{i, 2}, \cdots, v_{i, r_{i}}\right\}$. Let, $V=\left\{v_{1}, v_{2}, \cdots, v_{r}\right\}$ be the vertices, such that $v_{j} \in V, \forall j \in \mathcal{I}_{r}$, lies on the frontier of the set $\mathcal{R}$. The approximation of set $\mathcal{R}$ assuring the nonoverlapping and invariance characteristics is characterized by perturbing and positioning the frontier vertices sequentially. We start by defining the closed-loop mapping for any point in the set $\mathcal{R}$ preserving the invariance characteristics of the PWA controller:

$$
f_{\text {pwa }}(x)=A x+B u_{p w a}(x) \in \mathcal{R}
$$

From (14), we can represent the image of the set $\mathcal{R}$ preserving the invariance with,

$$
\mathcal{F}=\operatorname{Conv}\left\{f_{\text {pwa }}\left(v_{j}\right), \forall j \in \mathcal{I}_{r}\right\}
$$

In the reference [13], it has been shown that any sets of approximation of $\mathcal{R}$ denoted by $\mathcal{R}^{\alpha} \subseteq \mathcal{R}$ and $\mathcal{R}^{\alpha} \supseteq \mathcal{F}$, the invariance holds with respect to the approximated set $\mathcal{R}^{\alpha}$ for the existing PWA controller,

$$
\begin{gathered}
\mathcal{R}^{\alpha}=\operatorname{conv}\left\{v_{j}^{\alpha}=v_{j}+\Delta v_{j}, v_{i}^{\alpha}=v_{i}: \exists j \in \mathcal{I}_{r}, \forall i \in \mathcal{I}_{r} \backslash j\right. \\
\text { such that } \left.v_{j}^{\alpha} \in\left(\Psi^{v_{j}} \cap \mathcal{R}\right) \text { and } v_{j}^{\alpha} \notin \operatorname{int}(\mathcal{F})\right\}
\end{gathered}
$$

The validity of this construction will be demonstrated based on the idea in [13] and also considering the non-overlapping issues emerging from vertex perturbation. Figure 3 illustrates the set $\mathcal{R}$ with its image $\mathcal{F}$ and the vertex sensitivity sets for the corresponding vertices $v_{j}$ depicted with blue dots. In the following, we discuss about the possible scenarios where it leads to invalidation of invariance of the approximate set and overlapping issues.

Stage 1: From Figure 3, it can be noted that some boundary vertices of the image set $\mathcal{F}$ lies on the frontier of the set $\mathcal{R}$ and perturbing those vertices will result in violating the relation $\mathcal{R}^{\alpha} \supseteq \mathcal{F}$, here $\mathcal{R}^{\alpha}$ denotes the approximated set for the perturbed vertex. These vertices have no admissible perturbation in the first stage of the procedure. There after, we perturb any vertices that satisfies the relation $\mathcal{R}^{\alpha} \supseteq \mathcal{F}$.

Stage 2: Take two vertices $\left[v_{1}, v_{2}\right] \in V$, the vertex sensitivity of the set $\Psi^{v_{1}}$ for the vertex $v_{1}$ is computed assuming that the vertex $v_{2}$ is fixed and vice versa. Perturbing the vertex $v_{1}$ inside its vertex sensitivity set may invalidate the vertex sensitivity set computed for the vertex $v_{2}$. There after, we fix the position for the vertex after perturbation satisfying Stage 1 and recompute the set $\hat{\mathcal{R}}$ with its partition $\hat{\mathcal{R}}_{i}$, here the perturbed set is assumed to be $\mathcal{R}^{\alpha}$. We proceed to deriving the image set for the new $\mathcal{R}^{\alpha}$ and recompute the vertex sensitivity sets for all the untreated vertices.

Thus, we complete the proof that $\mathcal{R} \leftarrow \mathcal{R}^{\alpha}$ represents the approximation of the set $\mathcal{R}$ whose vertices are perturbed and positioned in a way that the set $\mathcal{R}^{\alpha}$ assures the nonoverlapping and the invariance property of the controller.

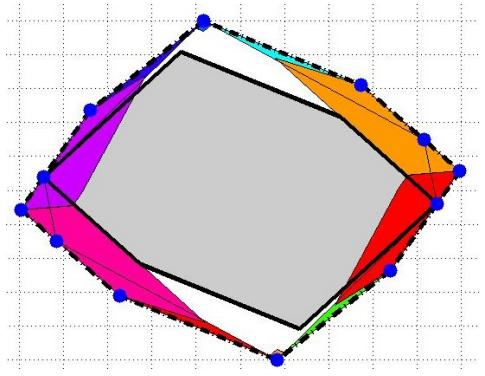

Fig. 3: Representation of the set $\mathcal{R}$ and its image $\mathcal{F}$ are represented by contour in dashed lines and the contour in full lines respectively. The colored polytopes apart from white and gray ones represent the vertex sensitivity for the vertices depicted in blue dots

Remark 1: Analyzing the PWA characteristics with respect to the vertex sensitivity for the boundary vertices are considered vital for many purposes. It could help the on-line PWA controller to tackle considerable perturbation on the system states. The most resourceful purpose is to perform "quantizer" operation on the state-space partition given by vertex representation. Such a process can effectively bring down the memory cost concerning the hardware requirements.

In the following we assume $\mathcal{R} \equiv \mathcal{R}^{\alpha}$ and we introduce a matrix $\bar{V} \in \mathbb{R}^{n \times p}$ to store all the vertices of all the polyhedral regions $\mathcal{R}_{i}, \forall i \in \mathcal{I}_{N}$ excluding the vertices on the boundary of the polyhedron $\mathcal{R}$,

$$
\bar{V}=\left[\bar{v}_{1}, \bar{v}_{2}, \cdots, \bar{v}_{p}\right]
$$

2) Treatment of one inner vertex for non-overlapping and invariance:

Definition 4: Consider the polyhedral partition $\mathcal{P}_{N}(\mathcal{R}) \subset$ $\mathbb{R}^{n}$ with $\mathcal{R}_{i}=\operatorname{Conv}\left\{v_{i, 1}, \cdots, v_{i, r_{i}}\right\}, i \in \mathcal{I}_{N}, \bar{V}=$ $\left[\bar{v}_{1}, \cdots, \bar{v}_{p}\right]$ and $\mathcal{R}$ is assumed to be invariant with respect to the closed-loop dynamics (1)-(4). For any $\bar{v} \in \mathcal{V}(\bar{V}) \in \mathbb{R}^{n}$, denote $\Lambda^{\bar{v}}$ as the subset of indexes of regions that satisfies $\left(\Psi^{\bar{v}} \cap \mathcal{R}_{j}\right) \neq \emptyset$ :

$$
\Lambda^{\bar{v}}=\left\{j \in \mathcal{I}_{N} \mid \bar{v} \in\left(\Psi^{\bar{v}} \cap \mathcal{R}_{j}\right) \neq \emptyset\right\}
$$

The set $\Upsilon^{\bar{v}} \subseteq \Psi^{\bar{v}} \subset \mathcal{R}$ is representing the invariancevertex sensitivity for a given vertex $\bar{v}$ if $\forall(\bar{v}+\Delta \bar{v}) \in \Upsilon^{\bar{v}}$, the following properties hold for the newly constructed polyhedral partition $\mathcal{P}_{N}(\hat{\mathcal{R}})$ :

1) $\hat{\mathcal{R}}$ is a polytope.

2) $\hat{\mathcal{R}}$ is invariant with respect to the closed loop dynamics.

3) $\hat{\mathcal{R}}=\cup_{i=1}^{N} \hat{\mathcal{R}}_{i}$.

4) $\operatorname{int}\left(\hat{\mathcal{R}}_{i}\right) \cap \operatorname{int}\left(\hat{\mathcal{R}}_{j}\right)=\emptyset, \forall i, j \in \mathcal{I}_{N}, i \neq j$.

Theorem 2: Let the subset of regions $\left\{\mathcal{R}_{j}: j \in \Lambda^{\bar{v}}\right\} \subset$ $\mathcal{P}_{N}(\mathcal{R})$ satisfy $\left(\Psi^{\bar{v}} \cap \mathcal{R}_{j}\right) \neq \emptyset$ and $\mathcal{R}$ be invariant with respect to the closed-loop dynamics (1)-(4). Then the invariance-vertex sensitivity for $\bar{v}$ is represented by a polyhedral set:

$$
\Upsilon^{\bar{v}}=\Psi^{\bar{v}} \cap\left\{\bar{v} \mid A \bar{v}+B\left(F_{j} \bar{v}+g_{j}\right) \in \mathcal{R}, \forall j \in \Lambda^{\bar{v}}\right\}
$$


Proof: Starting from the PWA state-feedback control assuring the invariance characteristics of the set $\mathcal{R}$,

$$
A \bar{v}+B\left(F_{i} \bar{v}+g_{i}\right) \in \mathcal{R}, \forall i \in \mathcal{I}_{N},
$$

and the vertex sensitivity described by the set $\Psi^{\bar{v}}$.

Now, locate the subset of regions of $\mathcal{P}_{N}(\mathcal{R})$ assuring the non-overlapping behavior for the vertex $\bar{v}$ and index it using a set $\Lambda^{\bar{v}}$. Using (20) and the description of $\Lambda^{\bar{v}}$, we are able to compute the sets denoted by $S_{j}^{\bar{v}}$ containing all the points for the PWA controllers, given by the indexes in the set $\Lambda^{\bar{v}}$, such that the invariance property is preserved. By simply intersecting the set $S_{j}^{\bar{v}}$ and $\Psi^{\bar{v}}$ sequentially we obtain the invariance-vertex sensitivity set:

$$
\Upsilon^{\bar{v}}=\bigcap_{j \in \Lambda^{\bar{v}}}\left(S_{j}^{\bar{v}} \cap \Psi^{\bar{v}}\right)
$$

\section{TREATMENT OF MULTIPLE VERTEX PERTURBATION}

In this section, we propose to perturb multiple vertices inside the polyhedral partition $\mathcal{P}_{N}(\mathcal{R})$. So far, we have discussed the perturbation of a single vertex assuring the nonoverlapping characteristics of the set $\mathcal{R}$ and the invariance property of the PWA closed-loop dynamics hold, under the assumption that all the other vertices are fixed.

We propose an algorithm to consider perturbing the position of all the inner vertices sequentially given in Algorithm 1 . The steps involved in transforming the set $\mathcal{R}$ to $\hat{\mathcal{R}}$ with respect to relocating the position of the vertices to the perturbed ones are described in the following.

- The first loop involvs the computation of vertex sensitivity, sensitivity margin and Chebyshev radius of the sensitivity margin set for all the inner vertices yet to be perturbed.

- Secondly, the the vertex, also called as candidate, that has the least fragility is selected. This is done by identifying the vertex that has the smallest Chebyshev radius associated to its invariance-vertex sensitivity.

- The next step is to consider a quantization function as $f(\bar{v})=\bar{v}+\Delta \bar{v}$, here $\Delta \bar{v}$ is a random vector satisfying $\|\Delta \bar{v}\|_{\infty} \leq 10^{-\epsilon}, \epsilon \in \mathbb{N}_{+}$.

- The regions that contain the perturbed (quantized) vertex are updated.

- The reconstruction of the set $\mathcal{R}$ is performed by removing the candidate vertex from the matrix $\bar{V}$ and restarting the same procedure for the remaining vertices.

\section{EXAMPLE}

Consider the discrete-time linear system,

$$
x_{k+1}=A x_{k}+B u_{k},
$$

where, $A=\left[\begin{array}{cc}1.4 & 0 \\ 1.8 & -1.1\end{array}\right]$ and $B=\left[\begin{array}{l}0.5 \\ 0.7\end{array}\right]$. The constraints on the states are $5 \leq\left[\begin{array}{ll}1 & 0\end{array}\right] x_{k} \leq-5$ and input constraint $5 \leq u_{k} \leq-5$. The weighing matrices $Q=\left[\begin{array}{ll}1 & 0 \\ 0 & 1\end{array}\right], R=1$ and prediction horizon of length 2 are considered in the finite time optimal control problem.

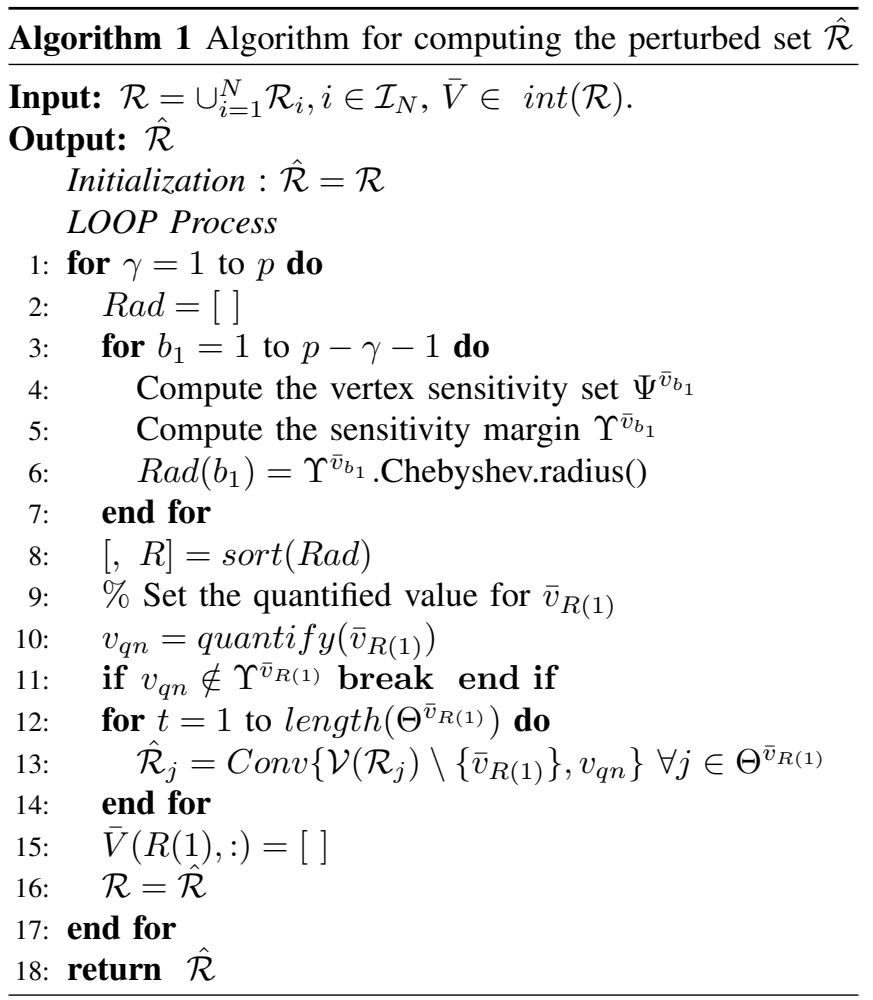

Solving the EMPC problem using MPT 3.0 toolbox [14] yields 13 affine controllers and its associated state space partitions. First, we approximated the set $\mathcal{R}$ by perturbing the vertices on the boundary of the set. A quantization function $f\left(v_{j}\right)=v_{j}+\Delta v_{j}, \forall j \in \mathcal{I}_{r}$ with a random variable $\left\|\Delta v_{j}\right\|_{\infty} \leq 10^{-2}$ is considered for perturbing the frontier vertices. A smaller quantizer function is chosen concerning the volume of the operating domain.

In the next step, we assume that the vertices on the boundary are fixed and we proceed to perturb the inner vertices in the set $\mathcal{R}$ sequentially as described in Algorithm 1. In the set $\mathcal{R}$ there are 8 inner vertices and we choose to manually displace them for this analysis for illustrative purpose. Figure 4 shows the functioning of the algorithm for each iteration. In the subplots from Figure 4, the polyhedral regions $\hat{\mathcal{R}}_{i}$ are presented with the vertex sensitivity and invariance-vertex sensitivity sets depicted in red and green color respectively, for the vertex that has the smallest Chebyshev radius. The symbols dot and $x$ in the subplots are the vertex candidate and the new position where the candidate needs to be pushed. The positions of the vertex candidate for each iteration and their new position are presented in the TABLE I. The regions that need to go under transformation for each iteration are also given in the table along with the Chebyshev radius for the candidate vertex. The numerical values of the vertices $\bar{v}$ are originally double precision representation but in the table we restricted the values till four decimal places due to space constraint. Starting from Figure 4 (a), for the first vertex candidate, we perturb the vertex to the position $[-4.0,1.6]^{T}$ there by affecting three regions with indexes $8,12,13$. The next subplot shows the new polyhedral regions after pertur- 
bation. After the 8th iteration, the subplot 4 (i) represents the final set $\hat{\mathcal{R}}$ that is the output of the algorithm 1 . In Figure 4 ,

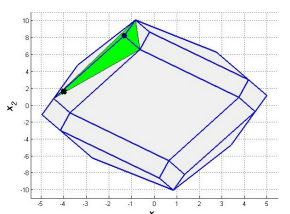

(a)

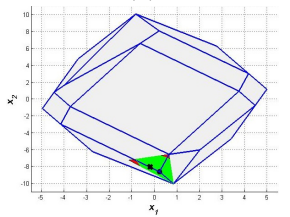

(d)

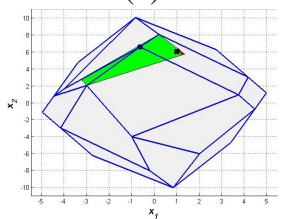

(g)

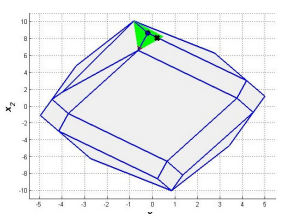

(b)

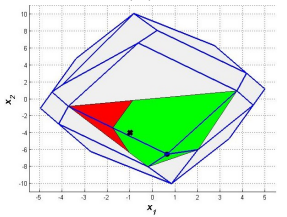

(e)

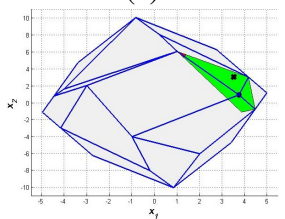

(h)

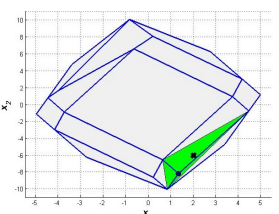

(c)

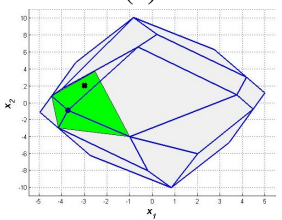

(f)

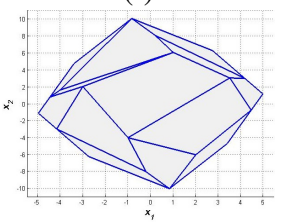

(i)
Fig. 4: In the subplots, the polyhedral regions $\hat{\mathcal{R}}_{i}$ are presented with the vertex sensitivity and invariant-vertex sensitivity sets depicted in red and green color respectively. The dot and the $x$ in the subplots are the vertex candidate and their new positions

it is obvious from the subplots that no overlapping took place although a very aggressive perturbation has been tested for illustration. This validates one part of our work. In order to conclude on the closed loop behavior, we simulated the state trajectories for the PWA controller for the outer vertices as initial states and present the results in Figure 5.

\begin{tabular}{|c|c|c|c|c|}
\hline$\gamma$ & $\bar{v}$ & $\begin{array}{c}\text { Chebyshev } \\
\text { radius }\end{array}$ & $\bar{v}+\Delta \bar{v}$ & $\Theta^{\bar{v}(1)}$ \\
\hline 1 & $\begin{array}{c}{[-1.3314,} \\
8.1440]^{T}\end{array}$ & 0.706 & {$[-4.0,1.6]^{T}$} & $\{8,12,13\}$ \\
\hline 2 & $\begin{array}{c}{[-0.2162,} \\
8.5668]^{T}\end{array}$ & 0.54 & {$[0.2,8]^{T}$} & $\{7,11,12\}$ \\
\hline 3 & $\begin{array}{c}{[1.3314,} \\
-8.1440]^{T}\end{array}$ & 0.706 & {$[2,-6]^{T}$} & $\{4,6,9\}$ \\
\hline 4 & $\begin{array}{c}{[0.2162,} \\
-8.5668]^{T}\end{array}$ & 0.70 & {$[-0.2,-8]^{T}$} & $\{1,3,4\}$ \\
\hline 5 & $\begin{array}{c}{[0.6361,} \\
-6.5235]^{T}\end{array}$ & 2.128 & {$[-1,-4]^{T}$} & $\{1,2,4,6\}$ \\
\hline 6 & $\begin{array}{c}{[-3.7291,} \\
-0.9076]^{T}\end{array}$ & 1.395 & {$[-3,2]^{T}$} & $\{1,2,5,8\}$ \\
\hline 7 & $\begin{array}{c}{[-0.6361,} \\
6.5235]^{T}\end{array}$ & 1.02 & {$[1,6]^{T}$} & $\{2,7,8,12\}$ \\
\hline 8 & $\begin{array}{c}{[3.7291,} \\
0.9076]^{T}\end{array}$ & 0.87 & {$[3.5,3]^{T}$} & $\{2,6,7,10\}$ \\
\hline
\end{tabular}

TABLE I: This table represents the vertex candidates for each iteration and their new position along with their Chebyshev radius. The last column shows the indexes of the subset of regions that are impacted by the perturbation of the vertex

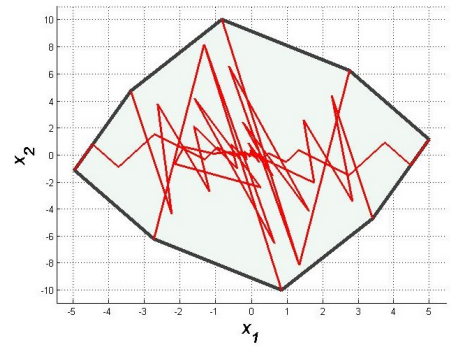

Fig. 5: The states trajectories for the polyhedral partition, outcome of the algorithm 1 as show in Fig 4 (i), for the vertices that lie on the boundary of the polytope.

\section{CONCLUSION}

In this work the analysis on the perturbation of the vertex representation has been presented for a PWA control function. The sensitivity set that preserve the closed-loop invariance in the event of perturbation has been computed. It was shown that a perturbed polyhedral partition can be constructed by treating sequentially each vertex with a higher priority on those with a small sensitivity margin.

\section{REFERENCES}

[1] A. Bemporard, M. Morrari, V. Dua and E. N. Pistikopoulos, The explicit linear quadratic regulator for constrained systems, Automatica, vol. 38(1), pp. 3-20, 2002.

[2] A. Alessio and A. Bemporad, A survey on explicit model predictive control, In Nonlinear model predictive control: Springer 2009, pp. 345-369.

[3] A. Grancharova, T. A. Johansen, Explicit Nonlinear Model Predictive Control Theory and Applications, Springer Science \& Business Media, vol. 429, 2012.

[4] T. A. Johansen, W. Jackson, R. Schreiber and P. Tondel, Hardware Synthesis of Explicit Model Predictive Controllers, IEEE Transactions on control systems technology, vol. 15, ,no. 1, Jan 2007.

[5] D. Ingole, M. Kvasnica, FPGA Implementation of Explicit Model Predictive Control for Closed Loop Control of Depth of Anesthesia, Preprints, 5th IFAC Conference on Nonlinear Model Predictive Control, Spain, September. 2015.

[6] G.Takács, G. Batista, M. Gulan, B. Rohal'-llkiv, Embedded explicit model predictive vibration control, Mechatronics, vol. 36, pp. 54-62, 2016.

[7] A. Suardi, S. Longo, E. C. Kerrigan and G. A. Constantinides, Robust explicit MPC design under finite precision arithmetic, 19th IFAC World Congress, vol. 47(3), pp. 2939-2944, 2014.

[8] S. Olaru, N. A. Nguyen, G. Bitsoris, P. Rodriguez-Ayerbe and M. Hovd, Explicit robustness margins for discrete-time linear systems with PWA control, System Theory, Control and Computing (ICSTCC), 2013 17th international Conference, pp. 380-385, Oct. 2013.

[9] N. A. Nguyen, S. Olaru, P. Rodrguez-Ayerbe, G. Bitsoris and M. Hovd, Explicit robustness and fragility margins for linear discrete systems with piecewise affine control law, Automatica, vol. 68, pp. 334-343, June, 2016.

[10] A. Knyazev, P. Zhu, S. Di Cairano, Explicit model predictive control accuracy analysis, 54th IEEE Conference on Decision and Control (CDC), Osaka, pp. 2389-2394, December. 2015.

[11] R. Koduri, S. Olaru and P. Rodriguez-Ayerbe, Sensitivity of Piecewise Control Laws with Respect to Perturbation of the State-Space Partition, 21st International Conference on Control Systems and Computer Science (CSCS), May, 2017.

[12] D. Q. Mayne, J. B. Rawlings, C. V. Rao, and P. O. M. Scokaert, Constrained model predictive control: Stability and optimality, Automatica, vol. 36(6), pp. 789-814, 2000.

[13] F. Scibilia, S. Olaru, M. Hovd, On feasible sets for MPC and their approximation, Automatica, vol. 47(1), pp. 133-139, January 2011.

[14] M. Herceg, M. Kvasnica, C. N. Jones and M. Morari, Multi-Parametric Toolbox 3.0, Proc. of the European Control Conference, pp. 502-510, July 2013. 